\title{
Téoros
}

Revue de recherche en tourisme

\section{Tourisme et aménagement du territoire au Maroc Quels agencements?}

\section{Saïd Boujrouf}

Volume 24, numéro 1, printemps 2005

Maroc, réalités et défis

URI : https://id.erudit.org/iderudit/1071104ar

DOI : https://doi.org/10.7202/1071104ar

Aller au sommaire du numéro

Éditeur(s)

Université du Québec à Montréal

ISSN

0712-8657 (imprimé)

1923-2705 (numérique)

Découvrir la revue

Citer cet article

Boujrouf, S. (2005). Tourisme et aménagement du territoire au Maroc : quels agencements ? Téoros, 24(1), 12-19. https://doi.org/10.7202/1071104ar d'utilisation que vous pouvez consulter en ligne.

https://apropos.erudit.org/fr/usagers/politique-dutilisation/ 


\section{Tourisme et aménagement du territoire au Maroc

\author{
Quels agencements?
}

\section{Saïd Boujrouf}

Ce thème, apparemment simple dans son intitulé bien que composé, pose toutefois trois grands problèmes au moins au géographe :

- Celui du rôle, de l'importance, de l'évolution et de la complexité de l'activité touristique dans la perspective du développement durable des territoires au Maroc.

- Celui du montage récent d'une politique globale d'aménagement du territoire dans un contexte de planification décentralisée et déconcentrée, de mondialisation et de primauté de l'individu contradictoires avec les exigences de l'État-Nation. Cela peut renforcer la complexité des relations entre les champs politique et économique dans leurs dimensions spatiales. Les interactions des champs politiques et économiques sont devenues de plus en plus intenses et la dimension spatiale prend davantage d'ampleur dans l'élaboration des stratégies du développement.

- Celui du rapport du tourisme à l'aménagement, du fait que, dans le contexte marocain, l'aménagement du territoire soit essentiellement une préoccupation des pouvoirs publics tandis que le tourisme est principalement l'affaire du secteur privé qui dépend de politiques publiques. II en résulte un flou persistant concernant des enjeux complexes, des acteurs nombreux et une marge de liberté fluctuante. La mise en cohérence des projets du territoire et la question même de la recomposition des territoires deviennent par conséquent problématiques.

La mise en tourisme pose, pour l'espace marocain, de nouvelles questions d'ordre spatial, économique, social, culturel et po-

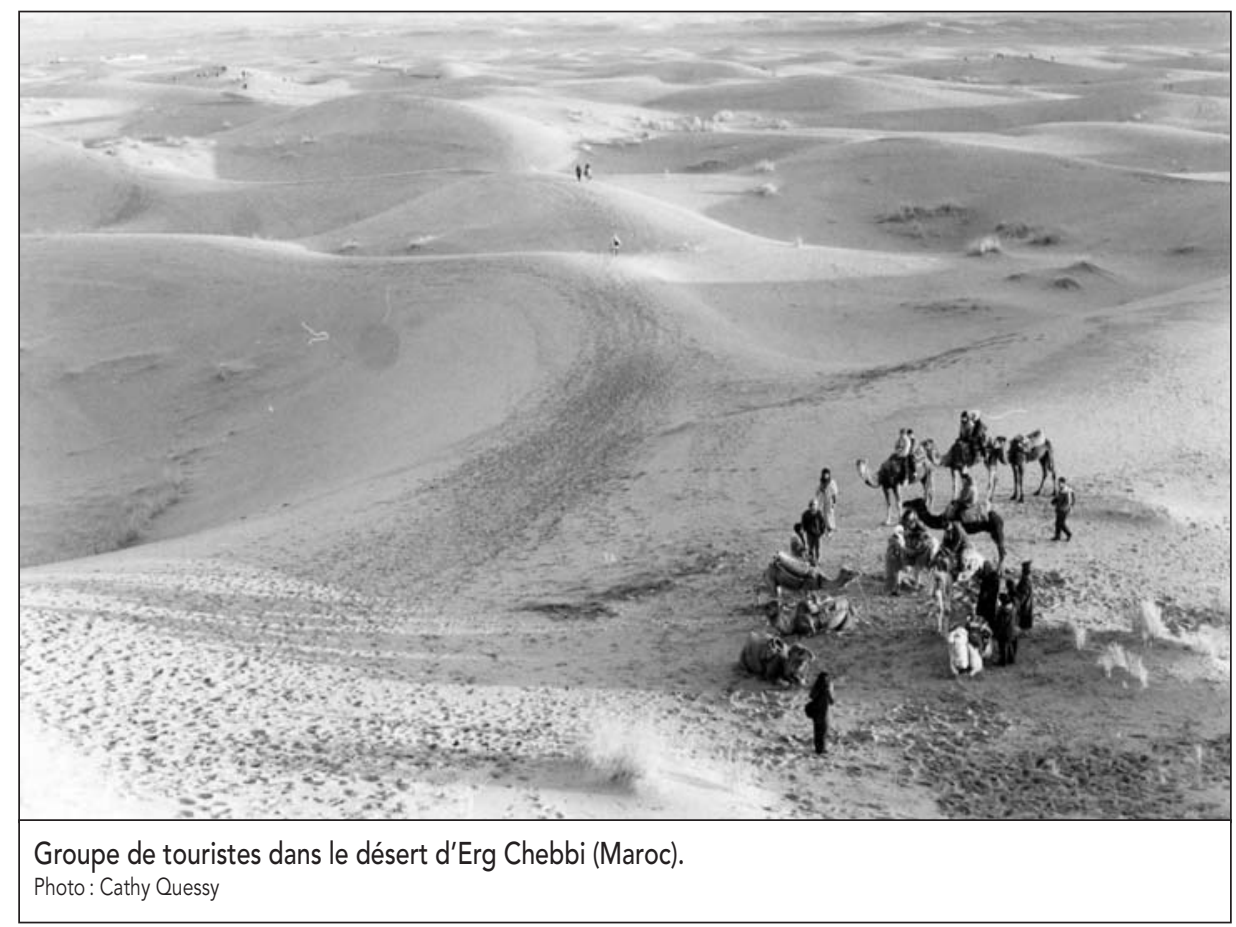

litique auxquelles il faudra dorénavant faire face. À la fin du siècle dernier, les préoccupations publiques dans ce secteur existaient déjà, bien avant le lancement des grands chantiers politiques de l'aménagement du territoire.

Comment alors l'aménagement du territoire au Maroc pourra-t-il intégrer le tourisme dans la stratégie globale de développement? Une telle politique est capable - sans aucun doute - de maîtriser la programmation de l'aménagement touristique, car l'équipement, le financement et le fonctionnement dépendent en grande partie de l'État et des collectivités territoriales. La réalité sur le terrain évolue rapidement. Les procédures lentes et les dispositifs administratifs - en cours d'innovation et de recomposition territoriales - poussent à s'interroger sur la place et le rôle du tourisme dans le système territorial marocain.

\section{Le tourisme au Maroc: émergence et édifications territoriales}

L'idée du projet touristique pour le Maroc n'émane pas d'un besoin exprimé par la société marocaine. Elle a été initiée par les Français à l'époque du protectorat (1912-1956) et ce, essentiellement pour répondre à une demande de résidents étrangers au Maroc.

Dans les années soixante, les organismes internationaux comme la Banque mondiale et l'Organisation des Nations unies encourageaient, eux aussi, les pays en voie de développement à adopter le tourisme comme moteur de développement socioécono- 
mique. De ce fait, le Maroc a demandé en 1964 aux experts de la Banque mondiale de faire un diagnostic territorial dans la perspective de lancer une politique économique qui ferait place au tourisme comme secteur économique à part entière.

Le Maroc a timidement démarré sa politique touristique en procédant à la « sectorialisation » du tourisme par la création du ministère du Tourisme en 1965, à l'annonce de l'activité touristique comme secteur économique prioritaire dans différents plans de développement national, principalement ceux de 1965-1967 et de 1968-1972 et, enfin, par l'encouragement des investisseurs touristiques à travers les codes d'investissement de 1973 et de 1983. L'État a ainsi tenu quatre rôles majeurs: celui de contrôleur, celui d'acteur promoteur porteur de projet, celui d'aménageur surtout dans les zones d'aménagement touristique et celui d'incitateur promulguant des mesures d'encouragement aux autres investisseurs.

Même si le tourisme représente pour les Marocains une activité plutôt occidentale en contraste avec une société arabo-musulmane traditionnelle, cela ne veut pas dire qu'il s'agisse d'un secteur marginal. Le tourisme devient un secteur stratégique pour le Maroc, malgré son coût énorme pour le budget public et privé qui s'élève à 25 milliards de dirhams depuis l'indépendance du pays (Alami, 2004 : 39). La part du tourisme dans les cinq plans publics de développement, allant de 1965 à 1985, varie de 1,8\% à 6,8 \% du budget public (Stafford, 1996 : 45), avec une moyenne de près de $5 \%$ par plan. L'investissement global prévu pour la période 2001-2010 dans le contratprogramme entre l'État et le secteur privé est de 80 milliards de dirhams. Le seul secteur hôtelier représente 30 milliards de dirhams pour la même période.

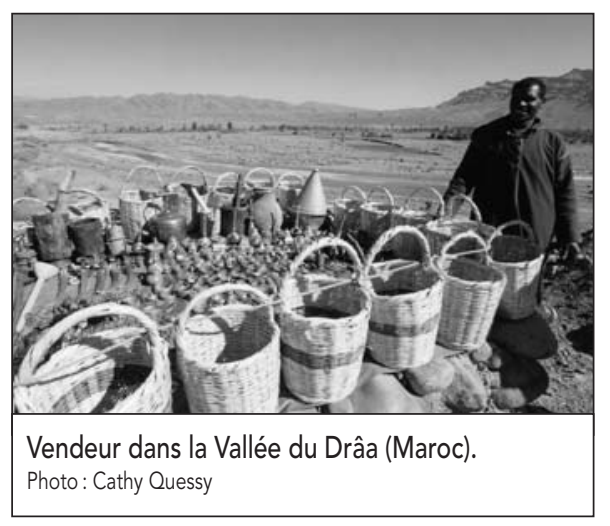

Ce secteur devient non seulement une source de devises ${ }^{1}$ et d'emplois, mais également un catalyseur d'innovation socio-économique, de communication (image du Maroc) et d'aménagement. Selon les estimations du ministère du Tourisme, le secteur du tourisme représente, dans sa globalité en 1998, 7 \% environ du produit intérieur brut, 608000 emplois directs et indirects (soit $5,8 \%$ de la population active occupée) et 16,5 milliards de dirhams de recettes en devises (Fédération du Tourisme, 2000 : 11). Lesdites recettes n'étaient en 1965 que de l'ordre de 332 millions de dirhams, soit, sur une période de 35 ans, une augmentation de $4900 \%$. La croissance des flux touristiques vers le Maroc explique en grand partie ce progrès fulgurant. Le nombre de touristes étrangers, qui n'était que de 17453 en 1964, a dépassé largement le total de 2,6 millions en 2002. Les Marocains Résidants à l'Étranger alimentent encore cette tendance par les visites annuelles qui ont dépassé en 2000 les 1,78 million d'arrivées².

L'évolution constante de l'infrastructure d'accueil se traduit par un dispositif territorial imposant, malgré son insuffisance. II est évident que le Maroc a pu réaliser un important parc d'hébergement ${ }^{3}$ puisqu'il est passé de 13000 lits classés en 1964 à 40732 lits en 1974, puis à 65134 lits en 1984, à 90511 en 1994, pour arriver à 97001 lits classés au début de 2002, soit une moyenne d'environs 2270 lits par an. Vu la traditionnelle division géographique du pays : un Maroc balnéaire opposé à celui de l'intérieur, l'infrastructure hôtelière globale estimée à 130668 lits (fin 2001), y compris les hôtels non classés, est inégalement répartie sur le territoire national au profit du balnéaire qui concentre $58 \%$. Par ailleurs, I'hébergement touristique au Maroc est plutôt urbain que rural : environ 62 \% des lits se concentrent dans les six grandes villes (Agadir, Marrakech, Casablanca, Tanger, Fès et Rabat). Ces villes offrent essentiellement deux grandes composantes de produits touristiques : la première est liée sous de multiples formes à la nature, notamment les mers méditerranéenne et atlantique, et la deuxième à la culture, notamment les villes impériales. Une diversification de produits se confirme de plus en plus à partir de ces grands pôles, avec la ville de Ouarzazate, pour développer un tourisme diffus dans quelques vallées des grands massifs montagneux et dans des oasis et des sites désertiques du sud intérieur marocain.
L'activité touristique concentre, en matière d'espace, les ressources humaines, les savoir-faire, les offres d'emploi et les équipements. De ce fait, le Maroc ne fait que confirmer, par sa longue expérience touristique, un déséquilibre territorial déjà remarquable. Quelles sont donc les causes d'une telle concentration qui préfère les grands pôles urbains et les côtes? Serait-ce un fait imposé par la recherche, de la part des aménageurs, des potentialités touristiques là où elles sont réelles et porteuses? Ne serait-ce pas, par ailleurs, une réponse à la demande internationale qui exige une telle offre spécifique à de tels lieux ? Ou encore, serait-ce le résultat de la politique touristique qui ne trouve pas encore son chemin pour s'articuler avec la politique d'aménagement du territoire national?

\section{Aménagement du territoire et aménagement touristique, quels dispositifs d'intégration territoriale?}

Dans plusieurs cas, l'aménagement touristique au Maroc est en fait une entreprise complexe dont on peut choisir le moment et le lieu de la réalisation sans toutefois pouvoir déterminer ses fins, ni même calculer les effets qui en résultent. Une telle situation s'expliquerait-elle par la prédominance de l'État dans l'aménagement touristique? Ou bien, les projets touristiques ne sont-ils pas un besoin exprimé par les populations locales et par ses représentations, ce qui provoquerait une éminente réticence de leur part? Ou encore serait-ce que le système de montage et de réalisation des projets de territoire est toujours en défaillance? Autrement dit : comment se fait concrètement l'aménagement touristique et dans quel environnement?

\section{Les Zones d'Aménagement Prioritaire (ZAP) d'avant la décentralisation de 1976}

La période allant de 1956, date de l'indépendance, à 1976, date de la promulgation de la plus importante charte communale, incarnait le grand espoir pour les Marocains de voir se réaliser de grands projets dans une perspective de développement et de modernisation. L'engagement d'une politique touristique a pris place dans le projet national. Sa mise en application est passée 
par le choix géographique de zones d'aménagement qui seraient essentiellement des lieux d'hébergement. La quantité de chambres-lits devenait le critère de base dans la concrétisation d'une politique dont l'État était le principal investisseur et développeur.

L'agencement spatial de l'aménagement touristique, se basant sur une démarche technique et sélective, produisait depuis 1965 une carte du Maroc des zones à aménager. II s'agissait de cinq zones d'aménagement touristique dont quatre sur le littoral et une seule à l'intérieur du pays : Tanger, Smir, Al Hoceima, Agadir, Grand-Sud et les circuits des villes impériales. De ce fait, les réalisations entreprises n'ont touché dans leur majorité que les zones côtières. Les organes de montage et d'application des projets étaient des sociétés publiques d'aménagement mises en place, en l'occurrence la Société Nationale d'Aménagement de la Baie de Tanger (SONABT), créée en 1967, et la Société Nationale d'Aménagement de la Baie d'Agadir (SONABA), créée en 1973 (figure 1). Agadir est devenu progressivement le premier pôle touristique du pays en termes d'hébergement, dans un temps record ne dépassant pas deux décennies. Sa capacité hôtelière est passée de 144 lits en 1964, soit de moins de $1 \%$, à plus de 13000 lits en 1983, soit plus de $20 \%$ des lits touristiques du Maroc ${ }^{4}$.

Ces aménagements localisés correspondaient à des impératifs, d'une part, faire face à la spéculation foncière qui commençait à se développer, surtout à Tanger, d'autre part, rétablir une ville, Agadir, qui venait de sortir d'un tremblement de terre dévastateur. Ces aménagements sélectifs ont probablement été conçus comme des points de diffusion touristique dans des espaces périphériques au sud et à l'intérieur du pays jusqu'alors pauvres en activités économiques.

Ce type d'agencement, devenu structurant au Maroc, n'était pas, en réalité, un moyen de régulation spatiale, mais un outil de répartition des projets économiques. Cette configuration territoriale a repositionné les acteurs et les espaces concernés par rapport à une logique de marché et non pas par un souci d'aménagement équilibré du territoire.
Parmi ces aménagements, les actions ont été menées de manière discontinue malgré l'implication éminente de l'administration publique; au début, le rythme de l'aménagement était irrégulier, à cause d'un démarrage difficile dû à un manque de compétence et de mécanismes adéquats. D'où "un constat d'insuffisances : manque de personnel qualifié, déficit des infrastructures de loisirs et de transport, mauvaise gestion des établissements hôteliers, modestie de la politique de promotion à l'étranger » (Alami, 2004 : 44).

La charte communale de 1976 a concrètement amené les communes sur la scène de l'aménagement touristique. Mais, malheureusement, elles se sont révélées incapables de mener des projets touristiques. Les comités techniques de coordination, provinciaux ou nationaux, dominés par le ministère de l'Intérieur, étaient eux seuls capables d'autoriser les projets touristiques. Les communes ont un rôle plus consultatif que décisionnaire, limité au fait d'autoriser les constructions et d'affecter le foncier municipal encore sous la tutelle de l'Autorité locale.

Le plan de développement 1978-1980 a provoqué un arrêt provisoire dans la politique d'aménagement touristique au Maroc, alors que le plan suivant (1981-1985) a lancé le début du désengagement de l'État de la production de lits touristiques sans pour autant perdre son rôle dans l'aménagement de l'espace touristique. Ce recul s'explique essentiellement par les effets négatifs de la crise pétrolière mondiale de 1979 et du conflit militaire dans le Sahara marocain.

\section{Les Zones d'Aménagement Touristique après la régionalisation de 1997 et le débat national sur l'aménagement du territoire}

\section{Décentralisation, régionalisation et aménagement du territoire}

Depuis l'ancien régime makhzanien jusqu'au régime de la monarchie constitutionnelle - d'avant la publication de la charte communale de 1976 -, en passant par le régime du protectorat, le Maroc a toujours et davantage renforcé sa tradition de développement centralisé. La régionalisation de 1997 va toutefois donner un coup de fouet à la décentralisation du développement au Maroc. La région détient dorénavant le sta- tut d'une collectivité territoriale pouvant engager son développement socioéconomique et culturel.

Rappelons que l'aménagement du territoire au Maroc souffre toujours d'un certain nombre de déficits malgré l'adoption du système de planification depuis l'indépendance. La Commission Mixte Interministérielle d'Aménagement du Territoire National, créée en 1968 (Bulletin officiel, 1968) ne s'est jamais réunie pour mettre en place une politique cohérente d'aménagement du territoire. L'administration chargée de l'aménagement du territoire était, depuis 1945, une division ou une direction qui n'avait pas de rattachement ministériel fixe. Ses préoccupations étaient focalisées principalement sur les questions d'urbanisme et d'habitat. Ce n'est qu'à la fin des années soixante que la problématique de l'aménagement de l'espace national est devenue un important champ d'investissement. En 1967, la direction de l'Aménagement du Territoire faisait partie du ministère de l'Intérieur et sa vision était plus large, dépassant le cadre de l'urbanisme. C'est en 1974 que le mariage entre l'aménagement du territoire et le tourisme a vu le jour dans un seul ministère pour les six ans qui ont suivi. L'administration a continué son déplacement parmi les ministères et le dernier en date est son rattachement en 2002 au ministère de l'Aménagement du Territoire, de l'Eau et de l'Environnement. L'absence d'un engagement clair pour une politique d'aménagement du territoire a ainsi retardé la production d'une loi sur l'aménagement du territoire durant plus de quatre décennies.

II a fallu attendre l'année 2000 pour que l'État lance un débat national sur l'aménagement du territoire. Cette nouvelle approche, introduisant la concertation entre les différents acteurs locaux et nationaux, a abouti en 2001 à l'élaboration de la première Charte Nationale de l'Aménagement du Territoire. Cette étape importante a été suivie, la même année, par la création du Conseil Supérieur d'Aménagement du Territoire (Bulletin officiel, 2001). Un Schéma National d'Aménagement du Territoire (SNAT) a pu voir le jour en 2002. Avec ces réalisations innovantes, on est passé d'une planification centralisée à une planification décentralisée qui intègre le tourisme comme un secteur clé dans le développement des territoires. 


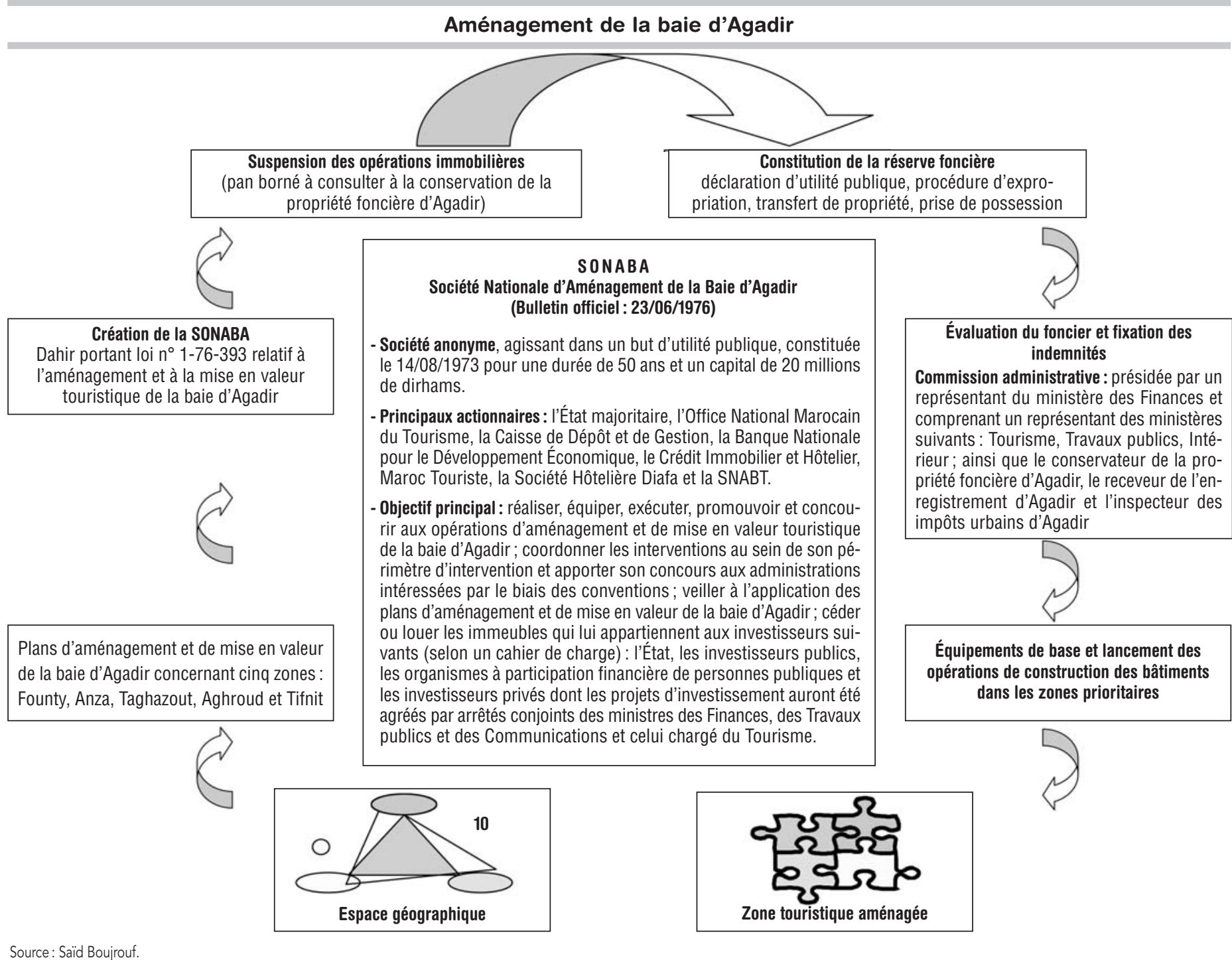

\section{L'aménagement touristique}

L'approche actuelle de la majorité des questions se fait de plus en plus par l'intégration de l'entreprise privée et de la "société civile ". Le maillage, les structures, les mobilisations publiques sont au service de ces nouveaux acteurs qui pourraient par la suite soutenir l'action publique en produisant des biens économiques et des services sociaux (Boujrouf, 2003). Les professionnels du tourisme, les entreprises et les associations ont réussi à négocier les nouveaux aménagements touristiques avec l'État dans un cadre contractuel et partenarial.

C'est pourquoi un important dispositif de déconcentration est mis en place $^{5}$ : les walis de région assurent au niveau de leur circonscription territoriale (Bulletin officiel, 2002) l'autorisation des projets d'investissement d'une valeur de moins de 200 millions de dirhams ${ }^{6}$, jusqu'ici prise par les pouvoirs centraux (figure 2). Ces décisions ont été déjà consolidées par la création, en 2002, de Centres Régionaux d'Investissement (C.R.I.), sorte de guichet unique réservé à la création d'entreprises et à la promotion de projets d'investissement. Quant aux projets dont le montant est supérieur à 200 millions de dirhams, ils bénéficient d'avantages spécifiques conventionnés, dans un cadre partenarial avec les pouvoirs centraux.

L'aménagement des zones touristiques « intégrées » reste, sur le plan financier, une affaire d'État, par le biais du Fond Hassan II pour le Développement Économique et Social, qui devra mettre à la disposition des investisseurs des terrains à vocation touristique. L'État procède par ailleurs à l'assouplissement des mécanismes de garantie pour simplifier les conditions de financement des activités touristiques. D'autres avantages, sous forme d'encouragements et d'aides financières, sont assurés par le code d'investissement.

L'aménagement touristique marque, pour cette nouvelle période, un grand retour de la mer par l'adoption du Plan Azur. II s'agit de soutenir la politique basée sur le tourisme balnéaire, suivie depuis les années soixante, tout en procédant à de remarquables innovations. La politique touristique marocaine n'est plus, comme avant, une 
proposition qui provient de l'étranger, mais un choix national pour soutenir l'économie nationale, l'entreprise marocaine et pour résorber les problèmes de chômage. Le développement du tourisme au Maroc est conditionné par l'importante croissance de la demande internationale du produit balnéaire: "plus de la moitié des européens (1 ${ }^{\text {er }}$ marché émetteur de touristes) voyageant vers la Grande Méditerranée sont attirés par le produit 'soleil et plage' "(Département du Tourisme, 2001). Le choix du balnéaire est justifié également par le fait que le Maroc dispose d'environ $3500 \mathrm{~km}$ de côtes atlantiques et méditerranéennes où Agadir est la seule grande ville touristique commercialisable à l'étranger.

L'étude de la stratégie d'aménagement touristique a identifié, sur le littoral, cinq nouvelles Zones d'Intérêt Touristique ${ }^{7}$, qui deviendront par la suite ce qu'on appelle déjà les Unités d'Aménagement Touristique (UAT). Les critères de choix sont principalement, et par ordre d'importance, la proximité d'un grand pôle urbain ou touristique, l'accessibilité routière portuaire aéroportuaire, la disponibilité foncière publique, l'existence d'un environnement favorable à la réalisation de projets touristiques de grande envergure.

Le processus de réalisation de l'aménagement des nouvelles stations touristiques passe d'abord par la sélection des sites favorables qui sont étudiés avec l'appui de la Banque mondiale pour approuver leur faisabilité. Un appel à manifestation d'intérêt est lancé ensuite par le Département du tourisme pour la présélection des aménageurs-développeurs privés. Ces grands opérateurs doivent proposer des scénarios d'aménagement en respectant le Data Room du Département du tourisme. Les aménageurs retenus signent enfin avec l'État les conventions d'investissement et les cahiers de charges. Ils pourront céder sur les sites aménagés des lots à des opérateurs hôteliers, des opérateurs de terrain de golf, des promoteurs immobiliers... (Département du tourisme, 2001). Les petits opérateurs touristiques, quant à eux, doivent passer par les dispositifs déconcentrés et décentralisés pour acquérir les terrains, avoir les autorisations, réaliser leurs projets et classer leurs établissements (figure 2).

\section{Figure 2}

Procédures et rouages administratifs pour la réalisation des projets touristiques au Maroc

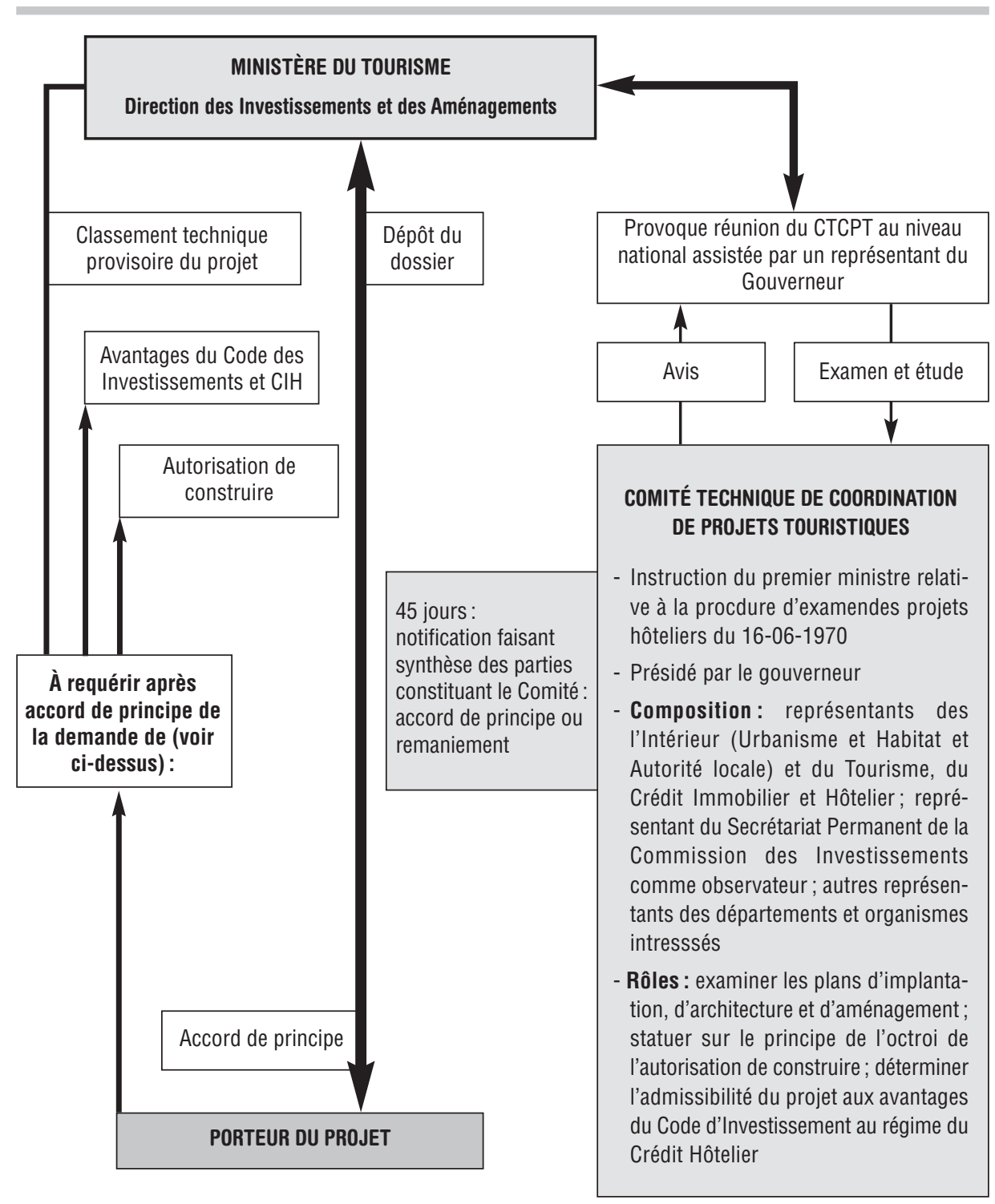

Source : Saïd Boujrouf.

\section{PROCÉDURE D’INVESTISSEMENT DE 1970}

II s'agit d'une nouvelle politique volontaire de développement et d'aménagement, politique basée sur une approche partenariale et contractuelle qui réunit l'État et les autres acteurs touristiques. Une convention-cadre et un contrat-programme 2001-2010 ont été signés entre l'État et la Fédération du Tourisme, filiale de la Confédération Générale des Entreprises Marocaines (CGEM). L'État s'engage à rendre l'offre plus compétitive et les projets plus rentables. Le secteur privé touristique s'engage pour sa part, en plus du développement des aménagements, à partici- per à la promotion de l'emploi, à contribuer à la formation, à augmenter la capacité d'hébergement, de l'investissement et de la promotion ${ }^{8}$.

Avec la signature de ce contrat, le domaine de l'aménagement touristique au Maroc n'est plus la préoccupation des seuls pouvoirs publics, mais plutôt le champ de participation d'une diversité d'acteurs dans le système territorial touristique marocain (Boujrouf, 2001). La pierre angulaire pour rentabiliser les opérations d'aménagement conçues et produites 


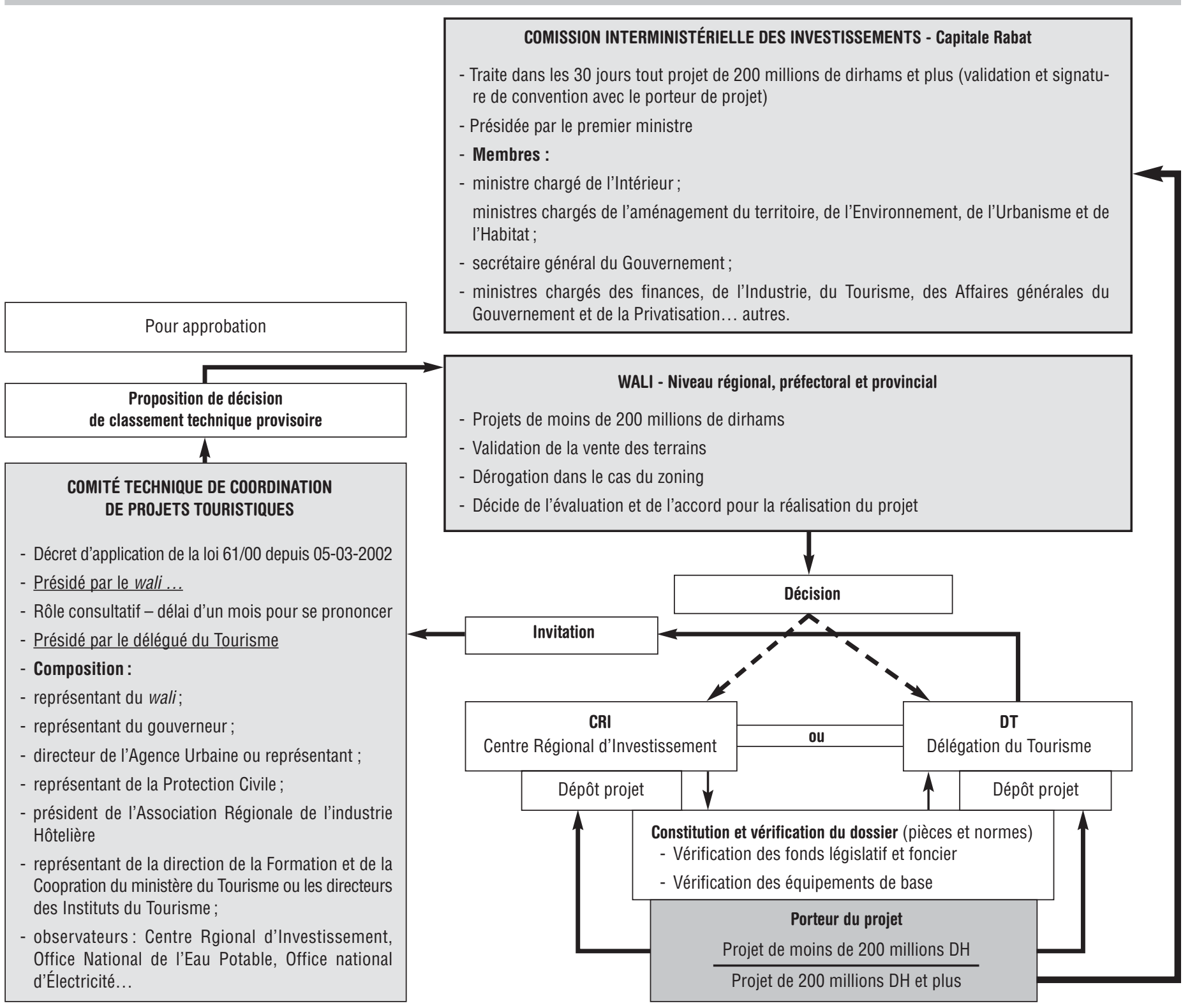

\section{PROCÉDURE D'INVESTISSEMENT À PARTIR DE 2002}

par l'État est l'entreprise touristique. La place du secteur privé se confirme de plus en plus par l'entrée en force des entrepreneurs marocains dans l'élaboration de la politique économique en général et touristique en particulier.

Malgré ces innovations, l'État reste certainement au centre de l'aménagement touristique du fait qu'il institutionnalise et planifie l'action, produit le foncier à des prix " compétitifs » allant jusqu'à $50 \%$ de sa valeur, oriente et produit les documents d'urbanisme, aménage / équipe, en partie par le biais des organes décentralisés (les collectivités locales) et déconcentrés (les walis), et assure enfin le contrôle et le suivi des travaux sur le terrain.

Comparé à celui de la période précédente, l'aménagement touristique étatique est en train de passer d'une logique du seul intérêt public à une nouvelle logique d'intérêt public-privé, donnant la priorité à la rentabilité et à l'efficacité de l'entreprise touristique. Une telle évolution devrait nécessairement se renforcer par la prise en charge des collectivités territoriales d'une bonne partie des aménagements touristiques, dans une perspective d'aménagement du territoire concerté et global.

Les collectivités locales tardent encore cependant à accomplir leur mission comme il se doit. Leur champ d'intervention reste limité au transfert foncier faisant partie de leur domaine privé, à autoriser les constructions, à réaliser quelques équipements de base et à récupérer les taxes fiscales. Ce rôle limité est-il dû à une gestion des projets d'aménagement touristique devenue extrêmement complexe 
Lits touristiques classés et non classés en 2002
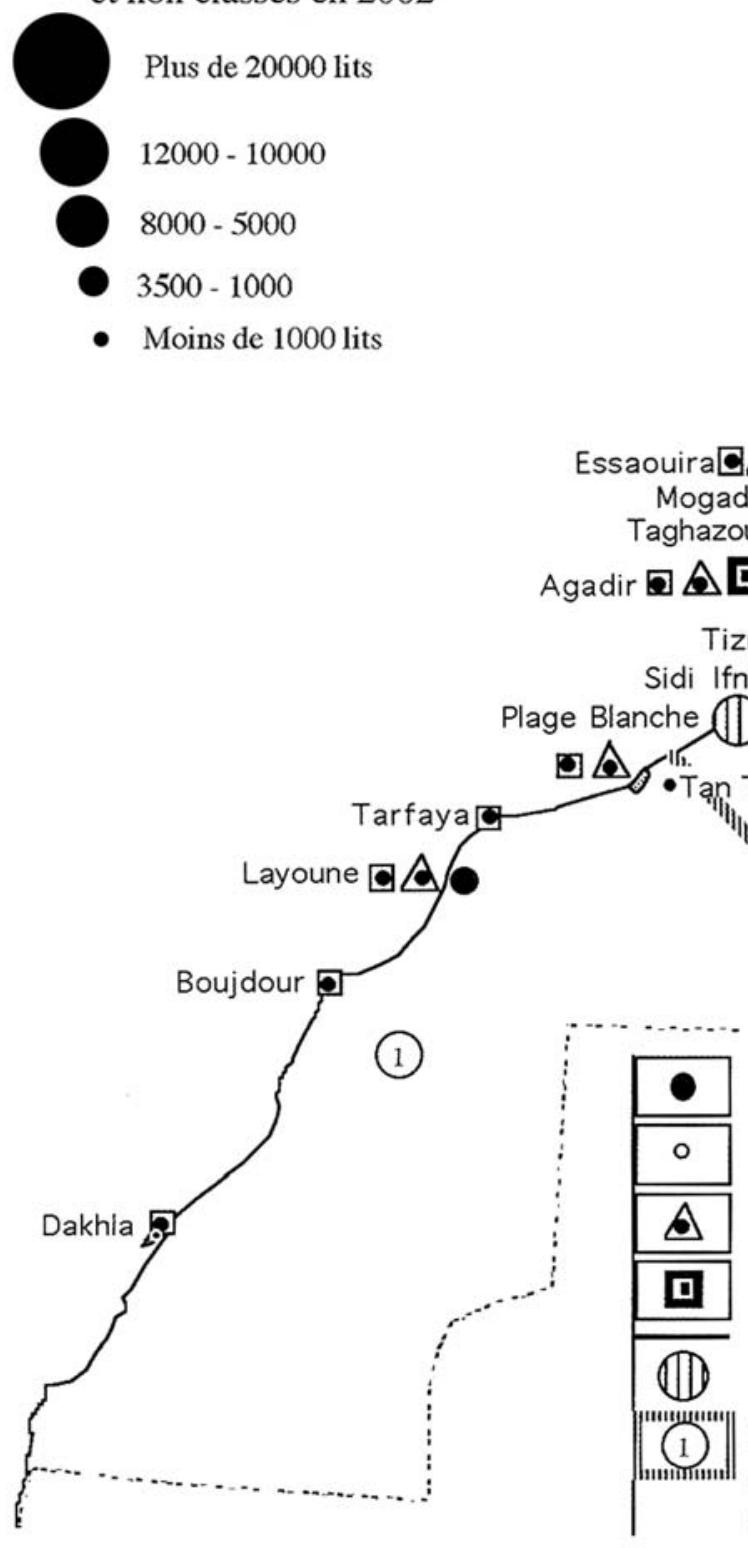

Source : Saïd Boujrouf.

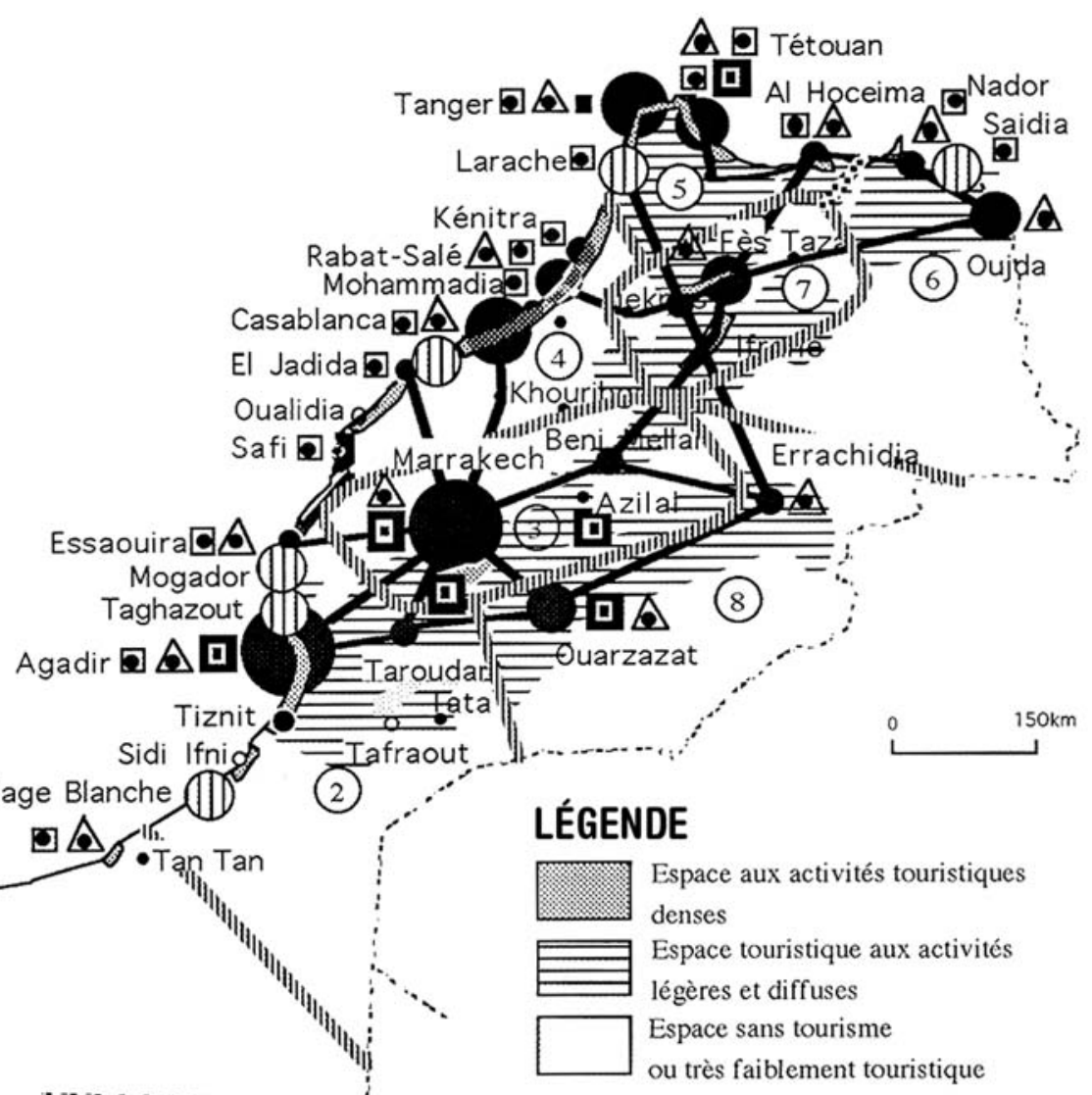

Ville à importants équipements touristiques

Petite localité touristique

\begin{tabular}{l|l} 
Aéroport & Port
\end{tabular}

Zone touristique d'intervention publique

Flux des principaux circuits touristiques du Maroc

Nouvelles Unités d'Aménagement touristique (Plan Azur-2010)

Limites des régions touristiques du découpage proposé par la "stratégie de développement du tourisme rural" (2002): 1-Laayoune et les régions sahariennes; 2-Agadir et sa région; 3-Marrakech et l'Atlas; 4-Rabat-Casablanca -El Jadida ou le Maroc atlantique; 5-Tanger-Tétouan-Larache ou la Méditerranée occidentale; 6-La Méditerranée orientale et le Marœ oriental; 7-Fès-Meknes et le Moyen Atlas; 8-Ouarzazate-Errachidia et le sud intérieur. et faisant appel à un niveau d'ingénierie rarement disponible à l'échelon local (Vlès, 2001: 61) ? Ou bien est-il lié au retard des jeunes collectivités territoriales à initier et à mener leurs politiques locales et régionales du tourisme en tant que chef d'orchestre d'aménagement et de développement?

\section{Conclusion}

La politique touristique au Maroc est antérieure à la politique d'aménagement du territoire, d'où les difficultés d'intégration et d'articulation du tourisme avec l'aménagement du territoire. Le montage des projets touristiques s'est fait, jusqu'à nos jours, par approches sectorielles et zonales, alors que l'approche territoriale est encore à l'état embryonnaire. II en résulte des espaces isolés et mal articulés avec leur environnement. L'exemple type est celui d'Agadir qui représente, dans plusieurs de ses composantes spatiales, un vrai ghetto touristique. 
Malgré la déconcentration récente de 2002 donnant aux walis de région un rôle clé dans les aménagements et les investissements touristiques, la création des stations touristiques demeure encore l'affaire des administrations centrales. Les documents d'urbanisme, les cessions foncières, les aides ou les exonérations d'impôts ou de taxes sont toujours approuvés à l'échelle de la capitale. L'aménagement se fait, par conséquent, du haut vers le bas, malgré quelques tentatives de lancer, dans le cadre de la stratégie de développement du tourisme rural, quelques projets à la base comme le cas des pays d'accueil touristique (OMT, 2002). Lesdits pays pourraient être des projets territoriaux montés à partir de l'échelon local.

L'État mise davantage sur le tourisme dans l'équilibre territorial de l'espace marocain (figure 3) du fait que l'activité touristique se développe généralement dans les arrièrespays et dans le Maroc périphérique (SNAT, 2002 : 127-128). C'est un argument pertinent, mais discutable lorsqu'il est comparé au degré de dégradation des environnements locaux (altération culturelle, problèmes de l'eau, de gestion des déchets...) et à ses faibles retombées socioéconomiques dans ces régions (Boujrouf, 1996), d'où le sentiment d'isolement et de marginalisation des populations locales. La politique touristique respecte sans aucun doute les critères d'ordre macroéconomique (l'offre touristique, l'importation des devises et la création d'emplois) plus que les critères du développement territorial qui font appel à la durabilité, à la justice sociale et à l'équilibre spatial. Ne s'agit-il pas en fait de concilier l'inconciliable (Merlin, 2001)?

Saïd Boujrouf est géographe et enseignant-chercheur à École doctorale internationale de tourisme, Laboratoire des études et de recherches sur les montagnes atlasiques (Département de Géographie), Faculté des lettres et sciences humaines de l'Université Cadi Ayyad de Marrakech.

\section{Notes}

1 Le tourisme devient, depuis 1999, le premier secteur financier dans la balance des paiements du Maroc avec 18746 millions DH (environ 2680 millions \$CAN) devant les transferts des Marocains Résidants à l'Étranger qui étaient de l'ordre de 18000 millions DH (en- viron 2571 millions \$CAN), par les recettes phosphatiques avec 13000 millions DH (environ 1857 millions $\$ C A N$ ), les ventes du textile avec 8000 millions DH (environ 1143 millions \$CAN), les produits agricoles avec 7200 millions DH (environ 1029 millions \$CAN) et la vente des produits de la mer: 6500 millions DH (environ 929 millions \$CAN). Source : Fédération du Tourisme, 2000 : 93.

2 Statistiques tirées de différents documents administratifs et des annuaires statistiques du Maroc, Direction de la Statistique, Rabat.

3 Statistiques tirées de différents documents administratifs et des annuaires statistiques du Maroc, Direction de la Statistique, Rabat. Statistiques du ministère du Tourisme, Rabat.

5 Ce dispositif se réfère à la Lettre Royale au premier ministre en date du 9 janvier 2002 relative à la gestion déconcentrée de l'investissement.

\section{Environ 28,57 millions \$CAN.}

7 II s'agit des zones de : 1) Saïdia-Ras El Ma sur la zone franche maroco-algérienne de la Méditerranée, d'une superficie de 600 hectares, ciblant les sports et loisirs ; 2) Khémis Sahel-Lixus près de la ville de Larache sur la côte atlantique nord, 299,5 hectares, pour devenir une station de bien-être, santé et nature ; 3) El Haouzia-Mazagan près de la ville d'El Jadida, à moins de 100 kilomètres de Casablanca, 476 hectares, pour être une destination «incentive» et sport de haut niveau ; 4) Mogador au sud de la ville d'Essaouira, 356 hectares, une destination pour mêler culture et sport nautique 5) Plage Blanche de Guélmim, 1000 hectares, pour les pratiques écotouristiques orientées vers la découverte et la remise en forme. Ces cinq stations balnéaires du plan Azur sont confortées par celle de Taghazoute (685 hectares) au nord d'Agadir avec un renforcement de Tanger. Le repositionnement des produits culturels de l'intérieur du Maroc vient consolider les aménagements touristiques par la création de Chérifia-Tasoultante (120 hectares) et d'Aguedal (96,27 hectares) à Marrakech et de l'Hippodrome à Fès. Le total de la superficie à aménager dans le cadre de la nouvelle politique touristique est estimé à environ 4000 hectares.

8 Le contrat prévoit à l'horizon 2010 la réception au Maroc de 10 millions de touristes par an, total qui ne dépassait en 2000 les 2,5 millions de touristes étrangers ; la construction de 80000 chambres nouvelles pour arriver à un total d'environ 128000 chambres homologuées; I'investissement hôtelier de 30 milliards de dirhams (environ 4,29 milliards \$CAN) et la création de 600000 emplois.

\section{Bibliographie}

Alami, Abdelhadi (2004), Le tourisme marocain, l'éternel espoir, Éditions Media Ten, 247 p.

Boujrouf, Saïd (1996), Tourisme et développement local, le cas de l'expérience de Tabant dans le Haut-Atlas central marocain, Actes du colloque international Quel avenir pour le tourisme en montagne au Maroc ?, Ministères de I'Intérieur et du Tourisme, Rabat, p. 63-76.

Boujrouf, Saïd (2001), « Acteurs et territoires touristiques de Marrakech », Lyon, Géocarrefour, $n^{\circ} 76$, p. 91-98.

Boujrouf, Saïd (2003), « Innovation et recomposition territoriale au Maroc, une mise en perspective géo-historique", dans Actes des rencontres de l'innovation territoriale publiés en ligne par UMR Pacte et Territoire, [www.iga.ujf-grenoble.fr/territoires/program mes/Innovation/PDF/12\%20BOUJROUF\% 20def.pdf] ; publié aussi sur le site [www. isiimm.agropolis.fr/].

Département d'Aménagement du Territoire (2002), Schéma National d'Aménagement du Territoire, les orientations, Rabat, DAT.

Département du Tourisme (2001), Plan Azur, Mémorandum conçu par le consortium CFG Group/Arthur Andersen agissant en qualité de conseiller du Département du Tourisme du Ministère de l'Économie, des Finances, de la Privatisation et du Tourisme.

Fédération du Tourisme (2000), Contrat programme 2000-2010, le tourisme: une vision, un défi, une volonté, Confédération Générale des Entreprises Marocaines.

Merlin, Pierre (2001), Tourisme et aménagement touristique, des objectifs inconciliables?, Paris, Les études de la Documentation Française.

Organisation mondiale du tourisme (2002), Stratégie de développement du tourisme rural, Madrid, Étude préparée pour le Ministère marocain de l'Économie, des Finances, de la Privatisation et du Tourisme.

Royaume du Maroc (1968), Bulletin officiel, Rabat, Imprimerie officielle, $\mathrm{n}^{\circ} 2911$.

Royaume du Maroc (2001), Bulletin officiel, Rabat, Imprimerie officielle, $n^{\circ} 4965$.

Royaume du Maroc (2002), Bulletin officiel, Rabat, Imprimerie officielle, $\mathrm{n}^{\circ} 4984$.

Stafford, Jean (dir.) (1996), Développement et tourisme au Maroc, Montréal, L'Harmattan.

Vlès, Vincent (2001), Service touristique local et aménagement du territoire, Paris, l'Harmattan. 